\title{
Type-1 Polarized Dendritic Cell-induced Antigen-specific Autologous Cytotoxic T Lymphocytes
}

National Cancer Institute

\section{Source}

National Cancer Institute. Type-1 Polarized Dendritic Cell-induced Antigen-specific

Autologous Cytotoxic T Lymphocytes. NCI Thesaurus. Code C128892.

A preparation of autologous cytotoxic T-lymphocytes (CTL), specifically reactive to melanoma-associated antigen 3 (MAGE-3), MAGE-4, survivin, human epidermal growth factor receptor 2 (HER2; ERBB2) and cyclooxyg enase-2 (COX-2), with potential immunomodulating activity. Peripheral blood mononuclear cells (PBMCs) are collected from the patient. Subsequently, autologous dendritic cells (DCs) are separated, treated with a certain combination of cytokines to produce polarized type-1 DCS (DC1), and then are loaded with MAGE-3/MAGE-4/survivin/HER2/COX-2 CTL epitope peptides. In turn, autologous CTLs are collected, exposed ex vivo to the antigen-loaded DC1s and subsequently expanded in vitro. Upon re-infusion of the DC1-induced MAGE-3/MAGE4/survivin/HER2/COX-2-specific autologous CT LS, the CT Ls target and lyse tumor cells expressing the tumor-associated antigens (TAAs). Exposure to DC1s generates more potent CTLs and thus induces a more potent CTL response against TAA-expressing tumor cells. The targeted TAAs play key roles in cellular proliferation and are overexpressed by a variety of cancer cell types. 